\title{
On Convergence of Monotone Finite Difference Schemes With Variable Spatial Differencing
}

\author{
By Richard Sanders
}

\begin{abstract}
Monotone finite difference schemes used to approximate solutions of scalar conservation laws have the advantage that these approximations can be proved to converge to the proper solution as the mesh size tends to zero. The greatest disadvantage in using such approximating schemes is the computational expense encountered since monotone schemes can have at best first order accuracy. Computation savings and effective accuracy could be gained if the spatial mesh were refined in regions of expected rapid solution variation.

In this paper we prove that standard monotone difference schemes, (satisfying a fairly unrestrictive CFL condition), converge to the "correct" physical solution even in the case when a nonuniform spatial mesh is employed.
\end{abstract}

1. Introduction. We consider the scalar conservation law

$$
u_{t}+\sum_{i=1}^{d} f_{i}(u)_{x_{i}}=0, \quad u(x, 0)=u_{0}(x),
$$

where $x \in \mathbf{R}^{d}$ and $t \geqslant 0$. Henceforth, we use the summation convention wherever convenient. This equation frequently arises as a model equation in continuum mechanics when the dissipative effects are neglected.

Classical solutions may not exist even for smooth initial data. A weak solution of (1.1) is a bounded measurable function such that for all $\varphi \in C_{0}^{\infty}\left(\mathbf{R}^{d} \times \mathbf{R}^{+}\right)$

$$
\int_{\mathbf{R}^{d} \times \mathbf{R}^{+}} u \varphi_{t}+f_{i}(u) \varphi_{x_{i}} d x d t=0, \quad \lim _{t \downarrow 0}\left\|u(x, t)-u_{0}(x)\right\|_{L^{1}\left(\mathbf{R}^{d}\right)}=0 .
$$

Solutions of (1.2) are not necessarily unique. For physical reasons, the limit solution of the viscous equation as viscosity tends to 0 is sought. This solution, assuming it exists, must satisfy for all $\varphi \in C_{0}^{\infty}\left(\mathbf{R}^{d} \times \mathbf{R}^{+}\right), \varphi \geqslant 0$ and all real numbers $c$

$$
-\int_{\mathbf{R}^{d} \times \mathbf{R}^{+}}|u-c| \varphi_{t}+\operatorname{sgn}(u-c)\left(f_{i}(u)-f_{i}(c)\right) \varphi_{x_{i}} d x d t \leqslant 0 .
$$

(1.3) is called the entropy condition. Solutions of (1.3) are called entropy solutions. Kružkov has shown in [5] that two solutions of (1.3) satisfy

$$
\left\|u\left(x, t_{1}\right)-v\left(x, t_{1}\right)\right\|_{L^{\prime}\left(\mathbf{R}^{d}\right)} \leqslant\left\|u\left(x, t_{0}\right)-v\left(x, t_{0}\right)\right\|_{L^{\prime}\left(\mathbf{R}^{d}\right)}
$$

for all $t_{1} \geqslant t_{0}$. Hence, condition (1.3) guarantees the uniqueness of solutions of (1.2). All classical solutions satisfy the entropy condition.

We extend the work of Crandall-Majda [1] and Kuznetsov [6]. We shall show how approximate solutions obtained from monotone finite difference schemes using

Received July 13, 1981.

1980 Mathematics Subject Classification. Primary 65M10, 65M15. 
nonuniform spatial differencing converge in the $L^{1}$ topology to the unique entropy solution of (1.2). We require essentially no restriction on the spatial grid other than it be a Cartesian product of partitioned coordinate axes. A sharp $L^{1}$ rate of convergence is also shown for these approximate solutions.

I would like to thank S. Osher for his gracious assistance during the preparation of this paper.

2.1. Definitions and Statements of Theorems. To simplify the analysis we restrict ourselves to two space dimensions. We lose no generality by doing this.

Partition $\mathbf{R}^{2}$ as follows:

$$
\mathbf{R}^{2}=\cup \Omega_{j, k},
$$

where $\Omega_{j, k}=\left[x_{j}, x_{j+1}\right) \times\left[y_{k}, y_{k+1}\right)$. As a measure of refinement call

$$
\delta=\max _{j, k}\left(\operatorname{diam}\left(B_{j, k}\right)\right)
$$

where $B_{j, k}$ is the smallest ball containing $\Omega_{j, k}$. Let $\Delta x_{j}=\left|x_{j+1}-x_{j}\right|$ and $\Delta y_{k}=$ $\left|y_{k+1}-y_{k}\right|$. Define the step function

$$
v_{\delta}(x, t)=u_{j, k}(t)
$$

when $x \in \Omega_{j, k}$.

Now consider the spatial difference operator:

$$
\begin{aligned}
D_{x}\left(F\left(v_{\delta}(x, t)\right)\right)= & \frac{1}{\Delta x_{j}} \Delta_{x}^{+} F_{1}\left(u_{j, k}(t), u_{j-1, k}(t)\right) \\
& +\frac{1}{\Delta y_{k}} \Delta_{y}^{+} F_{2}\left(u_{j, k}(t), u_{j, k-1}(t)\right)
\end{aligned}
$$

when $x \in \Omega_{j, k}$. We use the notation $\Delta_{x}^{+}, \Delta_{y}^{+}$to denote the forward difference operator in the $x, y$ direction. The numerical flux, $F_{i}(x, y), i=1,2$, is assumed to be locally Lipschitz continuous in both arguments. We require that $D_{x}\left(F\left(v_{\delta}\right)\right)$ be monotone and consistent, that is for $i=1,2$ :

1. $F_{i}(x, y)$ is nonincreasing in $x$,

2. $F_{i}(x, y)$ is nondecreasing in $y$,

3. $F_{i}(u, u)=f_{i}(u)$.

The initial data of (1.1) is discretized via the averaging operator $T_{\delta}$,

$$
T_{\delta}\left(u_{0}\right)(x) \equiv \frac{1}{\left|\Omega_{j, k}\right|} \int_{\Omega_{j . k}} u_{0}(s) d s
$$

when $x \in \Omega_{j, k}$. Throughout this paper we assume $u_{0}(x) \in L^{1} \cap L^{\infty} \cap B V$.

THEOREM I. (The method of lines.) The solutions of the infinite system of differential equations

$$
\frac{d v_{\delta}}{d t}+D_{x}\left(F\left(v_{\delta}\right)\right)=0, \quad v_{\delta}(x, 0)=T_{\delta}\left(u_{0}\right)(x),
$$

converge in $L^{\infty}\left(L^{1}\left(\mathbf{R}^{2}\right) ;[0, T]\right)$, as positive $\delta$ tends to 0 , to the unique entropy satisfying solution of (1.1). 
We may also discretize in time. Define $\Delta t^{n}=\left|t^{n+1}-t^{n}\right|$ and partition $\mathbf{R}^{2} \times \mathbf{R}^{+}$ as follows:

$$
\mathbf{R}^{2} \times \mathbf{R}^{+}=\bigcup_{j, k, n} \Omega_{j, k}^{n},
$$

where $\Omega_{j, k}^{n}=\Omega_{j, k} \times\left[t^{n}, t^{n+1}\right)$. As a measure of refinement call

$$
\delta=\max _{j, k, n}\left(\operatorname{diam}\left(B_{j, k}^{n}\right)\right) .
$$

Define the time difference operator

$$
D_{t}\left(v_{\delta}(x, t)\right)=\Delta_{t}^{+} u_{j, k}^{n} / \Delta t^{n}
$$

when $(x, t) \in \Omega_{j, k}^{n}$.

THEOREM II. (Fully implicit difference scheme.) The solutions of the difference scheme

$$
D_{t}\left(v_{\delta}(x, t)\right)+D_{x}\left(F\left(v_{\delta}\left(x, t^{n+1}\right)\right)\right)=0, \quad v_{\delta}(x, 0)=T_{\delta}\left(u_{0}\right)(x)
$$

converge in $L^{\infty}\left(L^{1}\left(\mathbf{R}^{2}\right) ;[0, T]\right)$, as positive $\delta$ tends to 0 , to the unique entropy satisfying solution of (1.1).

The explicit difference scheme poses a technical difficulty not yet encountered. The method of lines and the fully implicit difference scheme both have infinite domains of dependence. The explicit scheme does not. We therefore must attach an additional restriction on the size of the ratios, $\Delta t^{n} / \Delta x_{j}$ and $\Delta t^{n} / \Delta y_{k}$ to guarantee that the numerical domain of dependence contains the domain of dependence of the differential equation. This restriction is commonly called the CFL condition.

Define

$$
\Lambda^{x}=\max _{j, n}\left(\Delta t^{n} / \Delta x_{j}\right), \quad \Lambda^{y}=\max _{k, n}\left(\Delta t^{n} / \Delta y_{k}\right)
$$

and for $-\left\|u_{0}\right\|_{L^{\infty}} \leqslant x, y, w, z \leqslant\left\|u_{0}\right\|_{L^{\infty}}$ define

$$
H_{i}^{w, z}(x, y)=\left|F_{i}(x, w)-F_{i}(y, w)\right|+\left|F_{i}(z, x)-F_{i}(z, y)\right|
$$

for $i=1,2$. The CFL condition is equivalent to requiring that

$$
\Lambda^{x} H_{1}^{w_{1}, z_{1}}(x, y)+\Lambda^{y} H_{2}^{w_{2}, z_{2}}(x, y) \leqslant|x-y| \text {. }
$$

THEOREM III. (Explicit difference scheme.) Suppose (2.4) is satisfied uniformly as positive $\delta$ tends to 0 . Then the solutions of the difference scheme

$$
D_{t}\left(v_{\delta}(x, t)\right)+D_{x}\left(F\left(v_{\delta}\left(x, t^{n}\right)\right)\right)=0, \quad v_{\delta}(x, 0)=T_{\delta}\left(u_{0}\right)(x)
$$

converge in $L^{\infty}\left(L^{1}\left(\mathbf{R}^{2}\right) ;[0, T]\right)$, as positive $\delta$ tends to 0 , to the unique entropy satisfying solution of (1.1).

We also have an $L^{1}$ rate of convergence for these approximate solutions.

THEOREM IV. (Rate of convergence.) Let $v_{\delta}(x, t)$ be obtained from the schemes of Theorem I, Theorem II or Theorem III. Then for $T \geqslant t_{1} \geqslant t_{0} \geqslant 0$ we have

$$
\left\|u\left(x, t_{1}\right)-v_{\delta}\left(x, t_{1}\right)\right\|_{L^{1}\left(\mathbf{R}^{2}\right)} \leqslant\left\|u\left(x, t_{0}\right)-v_{\delta}\left(x, t_{0}\right)\right\|_{L^{1}\left(\mathbf{R}^{2}\right)}+K \sqrt{\delta},
$$

where $u(x, t)$ is the unique entropy satisfying solution of (1.1). $K$ depends linearly on the variation of the initial data and $\left|t_{1}-t_{0}\right|$. 
2.2. Examples. We show that two popular explicit finite difference schemes satisfy conditions (2.2) and (2.4). To simplify the analysis we assume the flux function, $f(u)$, is $C^{1}$ for all $|u| \leqslant\left\|u_{0}\right\|_{L^{\infty}}$.

The Lax-Friedrichs scheme utilizes the numerical flux

$$
F\left(u_{j}, u_{j-1}\right)=\frac{1}{2}\left(f\left(u_{j}\right)+f\left(u_{j-1}\right)\right)-\frac{1}{2 \Lambda}\left(u_{j}-u_{j-1}\right),
$$

where $\Lambda=\max _{j, n}\left(\Delta t^{n} / \Delta x_{j}\right)$. If

$$
\Lambda\left|f^{\prime}(u)\right| \leqslant 1
$$

for all $|u| \leqslant\left\|u_{0}\right\|_{L^{\infty}}$, then (2.2) is easily verified for (2.6). Also

$$
\begin{aligned}
\Lambda H^{w, z}(x, y)= & \Lambda / 2|(f(x)-f(y))-1 / \Lambda(x-y)| \\
& +\Lambda / 2|(f(x)-f(y))+1 / \Lambda(x-y)|,
\end{aligned}
$$

and (2.7) shows that

$$
\Lambda H^{w, z}(x, y)=\Lambda / 2(2 / \Lambda|x-y|)=|x-y| .
$$

The Engquist-Osher scheme utilizes the numerical flux

$$
F\left(u_{j}, u_{j-1}\right)=f^{-}\left(u_{j}\right)+f^{+}\left(u_{j-1}\right)
$$

where $f^{ \pm}$denotes the increasing, decreasing part of $f$-or more precisely

$$
\begin{aligned}
& f^{+}(u)=\int_{0}^{u} \max \left(f^{\prime}(s), 0\right) d s+f(0), \\
& f^{-}(u)=\int_{0}^{u} \min \left(f^{\prime}(s), 0\right) d s .
\end{aligned}
$$

One can easily verify that (2.8) satisfies (2.2). By virtue of (2.7) we also have that

$$
\begin{aligned}
\Lambda H^{w, z}(x, y) & =\Lambda\left(\left|f^{+}(x)-f^{+}(y)\right|+\left|f^{-}(x)-f^{-}(y)\right|\right) \\
& =\Lambda\left|\left(f^{+}(x)-f^{-}(x)\right)-\left(f^{+}(y)-f^{-}(y)\right)\right| \\
& \leqslant|x-y| .
\end{aligned}
$$

3. Preliminaries. In this section we state and prove a convergence theorem assuming the approximate solutions satisfy some basic inequalities. In Section 4, these inequalities are established for the schemes of Theorems I, II and III. We also prove two lemmas, Lemmas 3.3 and 3.4, which are fundamental in obtaining these estimates. The proofs of Lemmas 3.1 and 3.2 are given in Appendix II.

Let $\left\{v_{\delta}(x, t)\right\}$ be a family of approximate solutions. Suppose that

1. $\left\|v_{\delta}(x, t)\right\|_{L^{\prime}\left(\mathbf{R}^{d}\right)} \leqslant K_{1}$,

2. $\sup _{|h|>0}(1 /|h|)\left\|v_{\delta}(x+h, t)-v_{\delta}(x, t)\right\|_{L^{\prime}\left(\mathbf{R}^{d}\right)} \leqslant K_{2}$,

3. $\left\|v_{\delta}(x, t+\tau)-v_{\delta}(x, t)\right\|_{L^{\prime}\left(\mathbf{R}^{d}\right)} \leqslant K_{3}(|\tau|+\delta)$,

4. $\left\|v_{\delta}(x, t)\right\|_{L^{\infty}\left(\mathbf{R}^{d}\right)} \leqslant K_{4}$,

where $K_{1}$ through $K_{4}$ are constants independent of $\delta$ and $t \leqslant T$. Further suppose that for all $\varphi \in C_{0}^{\infty}\left(\mathbf{R}^{d} \times \mathbf{R}^{+}\right)$

5. $\lim _{\delta \rightarrow 0} \int_{\mathbf{R}^{d} \times \mathbf{R}^{+}} v_{\delta} \varphi_{t}+f_{i}\left(v_{\delta}\right) \varphi_{x_{i}} d x d t=0$,

and for $\varphi \geqslant 0$ and all real numbers $c$

6. $\lim _{\delta \rightarrow 0}-\int_{\mathbf{R}^{d} \times \mathbf{R}^{+}}\left|v_{\delta}-c\right| \varphi_{t}+\operatorname{sgn}\left(v_{\delta}-c\right)\left(f_{i}\left(v_{\delta}\right)-f_{i}(c)\right) \varphi_{x_{i}} d x d t \leqslant 0$. 
We then have

Proposition 3.1. Let $\left\{v_{\delta}(x, t)\right\}$ satisfy 1 through 6 above. Also suppose that $v_{\delta}(x, 0)$ tends to $u_{0}(x)$ in $L^{1}\left(\mathbf{R}^{d}\right)$ as $\delta$ tends to 0 . Then $\lim _{\delta \rightarrow 0} v_{\delta}(x, t)$ exists in $L_{\mathrm{loc}}^{1}\left(\mathbf{R}^{d}\right)$ on any bounded strip, $t \in[0, T]$, and the limit is the unique entropy satisfying weak solutions of

$$
u_{t}+f_{i}(u)_{x_{i}}=0, \quad u(x, 0)=u_{0}(x) .
$$

To prove Proposition 3.1 we use two lemmas.

LEMMA 3.1. Let $\left\{v_{\delta}(x, t)\right\}$ be an infinite family of scalar functions defined on $\mathbf{R}^{d} \times \mathbf{R}^{+}$, where $\delta$ is positive and tends to 0 . Suppose $\left\{v_{\delta}\right\}$ satisfies 1,2 , and 3 of Proposition 3.1. Then $\left\{v_{\delta}(x, t)\right\}$ contains a uniformly convergent subsequence in $L_{\text {loc }}^{1}\left(\mathbf{R}^{d}\right)$ on any bounded interval $[0, T]$.

Lemma 3.2. Suppose $\left\{v_{\delta}(x, t)\right\}$ satisfies 1 through 5 of Proposition 3.1. Further suppose that $v_{\delta}(x, 0)$ tends to $u_{0}(x)$ in $L^{1}\left(\mathbf{R}^{d}\right)$ as $\delta$ tends to 0 . Then every sequence of $\left\{v_{\delta}(x, t)\right\}$ has a subsequence converging in $L^{\infty}\left(L_{\mathrm{loc}}^{1}\left(\mathbf{R}^{d}\right) ;[0, T]\right)$ to a weak solution of (1.2).

Proof of Proposition 3.1. Lemma 3.1 and Lemma 3.2 show every sequence of $\left\{v_{\delta}(x, t)\right\}$ has a bounded convergent subsequence in $L^{\infty}\left(L_{\mathrm{loc}}^{1}\left(\mathbf{R}^{d}\right) ;[0, T]\right)$ to a weak solution of (1.2). This result, along with condition 6 of the proposition and the bounded convergence theorem, shows that the limit solution satisfies (1.3). (1.3) implies uniqueness. Therefore every sequence $\left\{v_{\delta}\right\}, \delta$ tending to 0 , has a convergent subsequence to a unique limit, proving every sequence converges.

It follows from Proposition 3.1 that the entropy satisfying solution of (1.2) must satisfy estimates 1 through 4.

LEMMA 3.3. Recall the definition of $T_{\delta}(u)$; see Eq. (2.3). If $v_{\delta}(x)=T_{\delta}(u)(x)$, we have

1. $\left\|v_{\delta}(x)\right\|_{L^{1}\left(\mathbf{R}^{d}\right)} \leqslant\|u(x)\|_{L^{1}\left(\mathbf{R}^{d}\right)}, v_{\delta}(x) \rightarrow u(x)$ in $L^{1}\left(\mathbf{R}^{d}\right)$ as $\delta \rightarrow 0$, and furthermore if $u(x) \in B V$.

2. $\operatorname{Var}\left(v_{\delta}(x)\right) \leqslant \operatorname{Var}(u(x))$.

Proof. We first prove 1. Using (2.3), we see that

$$
\left\|T_{\delta}(u)\right\|_{L^{1}} \leqslant \sum \int_{\Omega}\left(\frac{1}{|\Omega|} \int_{\Omega}|u(s)| d s\right) d x=\sum \int_{\Omega}|u(s)| d s=\|u\|_{L^{1}} .
$$

If $\omega \in C_{0}\left(\mathbf{R}^{d}\right)$, it is clear that $T_{\delta}(\omega)$ converges to $\omega$ in $L^{1}\left(\mathbf{R}^{d}\right)$ as $\delta$ tends to 0 . Therefore, since $T_{\delta}$ is linear,

$$
\begin{aligned}
\left\|T_{\delta}(u)-u\right\|_{L^{1}} & \leqslant\left\|T_{\delta}(\omega)-\omega\right\|_{L^{1}}+\left\|T_{\delta}(u-\omega)\right\|_{L^{1}}+\|\omega-u\|_{L^{1}} \\
& \leqslant\left\|T_{\delta}(\omega)-\omega\right\|_{L^{1}}+2\|\omega-u\|_{L^{1}} .
\end{aligned}
$$

The desired result is now immediate.

To prove 2 we lose no generality by assuming $d=2$. In this case it is easy to see that for fixed $y$ such that $y_{k} \leqslant y<y_{k+1}$

$$
\operatorname{Var}_{x}\left(v_{\delta}(x, y)\right) \Delta y_{k}=\sum_{j}\left|v_{\delta}\left(x_{j+1}, y\right)-v_{\delta}\left(x_{j}, y\right)\right| \Delta y_{k} .
$$


Using (2.3), this becomes

$$
\sum_{j}\left|\int_{y_{k}}^{y_{k+1}} \int_{0}^{1}\left(u\left(\xi_{j+1}, y\right)-u\left(\xi_{j}, y\right)\right) d t d y\right|,
$$

where

$$
\Delta y_{k}=\left|y_{k+1}-y_{k}\right|, \quad \xi_{j}=t x_{j+1}+(1-t) x_{j} .
$$

Using the triangle inequality and exchanging the order of summation and integration, we can bound the above quantity by

$$
\int_{y_{k}}^{y_{k+1}} \int_{0}^{1} \operatorname{Var}_{x}(u(x, y)) d t d y
$$

Summing Eqs. (3.1) and (3.2) on $k$, we obtain

$$
\int_{\mathbf{R}} \operatorname{Var}_{x}\left(v_{\delta}(x, y)\right) d y \leqslant \int_{0}^{1} \int_{\mathbf{R}} \operatorname{Var}_{x}(u(x, y)) d y d t=\int_{\mathbf{R}} \operatorname{Var}_{x}(u(x, y)) d y .
$$

A symmetric argument completes the proof.

LEMMA 3.4. Suppose $v_{\delta}(x)$ is a piecewise constant function on $\mathbf{R}^{d}$ defined by $v_{\delta}(x)=u_{j_{1}, \ldots, j_{d}}$ when $x \in \Omega_{j_{1}, \ldots j_{d}}$. Then

$$
\sup _{|h|>0} \frac{1}{|h|}\left\|v_{\delta}(x+h)-v_{\delta}(x)\right\|_{L^{1}\left(\mathbf{R}^{d}\right)} \leqslant \operatorname{Var}\left(v_{\delta}(x)\right) \text {. }
$$

Proof. It again suffices to assume $d=2$. Let $\varepsilon_{x}=\inf \left(\Delta x_{j}\right)$ where $\Delta x_{j}=$ $\left|x_{j+1}-x_{j}\right|$. For any positive number $h$ we can set

$$
h=(l+\theta) \varepsilon_{x},
$$

where $l$ is an integer and $0 \leqslant \theta<1$. Using the triangle inequality, we have

$$
\begin{aligned}
\int_{\mathbf{R}} \mid v_{\delta}(x+h, y) & -v_{\delta}(x, y) \mid d x \\
\leqslant & l \int_{\mathbf{R}}\left|v_{\delta}\left(x+\varepsilon_{x}, y\right)-v_{\delta}(x, y)\right| d x \\
& +\int_{\mathbf{R}}\left|v_{\delta}\left(x+\theta \varepsilon_{x}, y\right)-v_{\delta}(x, y)\right| d x .
\end{aligned}
$$

Now observe that for $y_{k} \leqslant y<y_{k+1}$

$$
\begin{aligned}
\int_{x_{j}}^{x_{j+1}} & \left|v_{\delta}\left(x+\theta \varepsilon_{x}, y\right)-v_{\delta}(x, y)\right| d x \\
& =\int_{x_{j}}^{x_{j+1}-\theta \varepsilon_{x}}\left|u_{j, k}-u_{j, k}\right| d x+\int_{x_{j+1}-\theta \varepsilon_{x}}^{x_{j+1}}\left|u_{j+1, k}-u_{j, k}\right| d x \\
& =\theta \varepsilon_{x}\left|u_{j+1, k}-u_{j, k}\right| .
\end{aligned}
$$

Using this identity, (3.3) becomes

$$
\int_{\mathbf{R}}\left|v_{\delta}(x+h, y)-v_{\delta}(x, y)\right| d x \leqslant(l+\theta) \varepsilon_{x} \sum_{j}\left|u_{j+1, k}-u_{j, k}\right| .
$$


Multiplying this by $\Delta y_{k}$ and summing on $k$, we obtain

$$
\begin{aligned}
& \int_{\mathbf{R}^{2}}\left|v_{\delta}(x+h, y)-v_{\delta}(x, y)\right| d x d y \\
& \leqslant h \sum_{j, k}\left|u_{j+1, k}-u_{j, k}\right| \Delta y_{k}=h \int_{\mathbf{R}} \operatorname{Var}_{x}\left(v_{\delta}(x, y)\right) d y .
\end{aligned}
$$

Treating $y$ in a similar manner gives

$$
\begin{aligned}
& \int_{\mathbf{R}^{2}}\left|v_{\delta}\left(x+h_{1}, y+h_{2}\right)-v_{\delta}(x, y)\right| d x d y \\
& \quad \leqslant\left\|h_{1} \hat{e}_{x}\right\| \int_{\mathbf{R}} \operatorname{Var}_{x}\left(v_{\delta}(x, y)\right) d y+\left\|h_{2} \hat{e}_{y}\right\| \int_{\mathbf{R}} \operatorname{Var}_{y}\left(v_{\delta}(x, y)\right) d x \\
& \quad \leqslant\left\|h_{1} \hat{e}_{x}+h_{2} \hat{e}_{y}\right\| \operatorname{Var}\left(v_{\delta}(x, y)\right) .
\end{aligned}
$$

Dividing by $\left\|h_{1} \hat{e}_{x}+h_{2} \hat{e}_{y}\right\|$ and taking the supremum over $|h|>0$ gives the desired result.

4.1. Proof of Theorem I. For a fixed mesh, the long time existence and uniqueness of the solution of

$$
\frac{d v_{\delta}}{d t}+D_{x}\left(F\left(v_{\delta}\right)\right)=0, \quad v_{\delta}(x, 0)=T_{\delta}(u)
$$

is easily obtained by Picard iteration and the following maximum principle.

I. Maximum Principle.

$$
\min \left(v_{\delta}(x, 0)\right) \leqslant v_{\delta}(x, t) \leqslant \max \left(v_{\delta}(x, 0)\right)
$$

Proof. For fixed $t$ let $m=\max \left(u_{j, k}(t)\right)$. Using (2.1) along with conditions (2.2), we find that $d m / d t \leqslant 0$. This shows the maximum of $v_{\delta}(x, t)$ is nonincreasing. A similar argument shows the minimum of $v_{\delta}(x, t)$ is nondecreasing.

As well as showing long time existence and uniqueness this gives 4 of Proposition 3.1. Next, we verify that the other conditions of Proposition 3.1 are satisfied by $v_{\delta}(x, t)$.

II. $L^{1}$ Boundedness. To show 1 of Proposition 3.1 multiply (4.1) by $\Delta x_{j}, \Delta y_{k}$ and $\psi_{j, k}$ where $\psi_{j, k}=\operatorname{sgn}\left(u_{j, k}(t)\right)$. Sum on $j$ to obtain

$$
\frac{d}{d t} \sum_{j}\left|u_{j, k}\right| \Delta x_{j} \Delta y_{k}=-\sum_{j} \Delta_{x}^{+} F_{1}(\cdot, \cdot) \psi_{j, k} \Delta y_{k}-\sum_{j} \Delta_{y}^{+} F_{2}(\cdot, \cdot) \psi_{j, k} \Delta x_{j}
$$

which is true in the sense of distributions. Since $\Delta_{x}^{+} F_{1}(0,0)=0$, we have

$$
-\sum_{j} \Delta_{x}^{+} F_{1}(\cdot, \cdot) \psi_{j, k}=-\sum_{j} \Delta_{x}^{+}\left(F_{1}(\cdot, \cdot)-F_{1}(0,0)\right) \psi_{j, k} \text {. }
$$

Summing by parts and adding and subtracting $F_{1}\left(0, u_{j-1, k}\right)$, this becomes

$$
\begin{aligned}
\sum_{j}\left(F_{1}\left(u_{j, k}, u_{j-1, k}\right)\right. & \left.-F_{1}\left(0, u_{j-1, k}\right)\right)\left(\psi_{j, k}-\psi_{j-1, k}\right) \\
& +\sum_{j}\left(F_{1}\left(0, u_{j-1, k}\right)-F_{1}(0,0)\right)\left(\psi_{j, k}-\psi_{j-1, k}\right) .
\end{aligned}
$$

Each term of the above sums can be seen to be nonpositive by virtue of 1 and 2 of (2.2). Therefore the first term on the right-hand side of (4.2) is nonpositive. 
Eliminate this term, sum the result on $k$ and repeat the same argument to obtain

$$
\frac{d}{d t}\left\|v_{\delta}(x, t)\right\|_{L^{\prime}}=\frac{d}{d t} \sum_{j, k}\left|u_{j, k}\right| \Delta x_{j} \Delta y_{k} \leqslant 0 \text {. }
$$

This estimate along with Lemma 3.3 shows

$$
\left\|v_{\delta}(x, t)\right\|_{L^{1}} \leqslant\left\|u_{0}(x)\right\|_{L^{1}}
$$

for all $t \geqslant 0$.

III. Variation Boundedness. This is the single most important estimate we obtain. At no time is the solution assumed to be translation invariant. Hence, a similar argument applies with few modifications to problems in which the flux functions are explicit functions of $x$ and $t$.

To obtain 2 of Proposition 3.1 one should observe that

$$
\frac{d}{d t} \Delta_{x}^{+} u_{j, k}=-\Delta_{x}^{+}\left(\frac{1}{\Delta x_{j}} \Delta_{x}^{+} F_{1}(\cdot, \cdot)+\frac{1}{\Delta y_{k}} F_{2}(\cdot, \cdot)\right) \text {. }
$$

Multiply this by $\Delta y_{k}$ and $\psi_{j, k}$ where $\psi_{j, k}=\operatorname{sgn}\left(u_{j+1, k}-u_{j, k}\right)$. Summing on $j$, we have

(4.3) $\frac{d}{d t} \sum_{j}\left|\Delta_{x}^{+} u_{j, k}\right| \Delta y_{k}=-\sum_{j} \Delta_{x}^{+}\left(\frac{\Delta y_{k}}{\Delta x_{j}} \Delta_{x}^{+} F_{1}(\cdot, \cdot)+\Delta_{y}^{+} F_{2}(\cdot, \cdot)\right) \psi_{j, k}$.

Summing by parts, the right-hand side of (4.3) becomes

$$
\sum_{j} \frac{\Delta y_{k}}{\Delta x_{j}} \Delta_{x}^{+} F_{1}(\cdot, \cdot)\left(\psi_{j, k}-\psi_{j-1, k}\right)-\sum_{j} \Delta_{x}^{+} \Delta_{y}^{+} F_{2}(\cdot, \cdot) \psi_{j, k} \text {. }
$$

As before

$$
\Delta_{x}^{+} F_{1}(\cdot, \cdot)\left(\psi_{j, k}-\psi_{j-1, k}\right) \leqslant 0
$$

So we see the first term of (4.4) is nonpositive. Sum on $k$ and make a similar observation on the second term to obtain

$$
\frac{d}{d t} \sum_{j, k}\left|\Delta_{x}^{+} u_{j, k}\right| \Delta y_{k} \leqslant 0
$$

A symmetric argument shows

$$
\frac{d}{d t} \sum_{j, k}\left|\Delta_{y}^{+} u_{j, k}\right| \Delta x_{j} \leqslant 0 .
$$

This, together with Lemma 3.3, shows

$$
\operatorname{Var}\left(v_{\delta}(x, t)\right) \leqslant \operatorname{Var}\left(u_{0}(x)\right) \text {. }
$$

Using Lemma 3.4 completes the proof.

IV. Continuity in Time. For any conservation law with Lipschitz continuous fluxes, Lipschitz continuity in time follows immediately from variation boundedness. We see this for the method of lines by integrating (4.1) from $t$ to $t+\tau$. Since $F_{i}(x, y)$ is Lipschitz continuous, the triangle inequality gives us

$$
\begin{aligned}
& \left|v_{\delta}(x, t+\tau)-v_{\delta}(x, t)\right| \Delta x_{j} \Delta y_{k} \\
& \qquad K \sum_{i=0}^{1} \int_{t}^{t+|\tau|}\left(\left|\Delta_{x}^{+} u_{j-i, k}\right| \Delta y_{k}+\left|\Delta_{y}^{+} u_{j, k-i}\right|\right) \Delta x_{j} d t .
\end{aligned}
$$

Summing on $j$ and $k$ gives condition 3 of Proposition 3.1. 
V. Weak Solution. The proof of 6 of Proposition 3.1 is similar to the proof of the Lax-Wendroff theorem, [8], and will be omitted.

VI. Entropy Solution. To show 7 of Proposition 3.1 we make use of the inequality

$$
\Delta_{x}^{+}\left[\left(F_{1}(\cdot, \cdot)-F_{1}(c, c)\right) \psi_{j, k}\right] \leqslant \Delta_{x}^{+}\left(F_{1}(\cdot, \cdot)-F_{1}(c, c)\right) \psi_{j, k}
$$

where $\psi_{j, k}=\operatorname{sgn}\left(u_{j, k}-c\right)$. Using this along with (4.1) gives

$$
\begin{aligned}
\frac{d}{d t}\left|u_{j, k}-c\right| \Delta x_{j} \Delta y_{k}+\Delta_{x}^{+}\left[\left(F_{1}(\cdot, \cdot)-F_{1}(c, c)\right) \psi_{j, k}\right] \Delta y_{k} \\
+\Delta_{y}^{+}\left[\left(F_{2}(\cdot, \cdot)-F_{2}(c, c)\right) \psi_{j, k}\right] \Delta x_{j} \leqslant 0 .
\end{aligned}
$$

The remainder of the proof is identical to a continuous time version of the proof of the Lax-Wendroff theorem.

This completes the proof of Theorem I.

4.2. Proofs of Theorems II and III. For the fully implicit scheme the proofs of properties 4 through 6 of Proposition 3.1 are routine and will be omitted. Properties 1 and 2 are immediate from the proofs of Section 4.1 by noting that

$$
\begin{aligned}
& \sum_{j, k}\left|u_{j, k}^{n+1}\right| \Delta x_{j} \Delta y_{k}=\sum_{j, k} u_{j, k}^{n+1} \psi_{j, k}^{n+1} \Delta x_{j} \Delta y_{k} \\
& \leqslant \sum_{j, k} u_{j, k}^{n} \psi_{j, k}^{n+1} \Delta x_{j} \Delta y_{k} \leqslant \sum_{j, k}\left|u_{j, k}^{n}\right| \Delta x_{j} \Delta y_{k}
\end{aligned}
$$

where $\psi_{j, k}^{n+1}=\operatorname{sgn}\left(u_{j, k}^{n+1}\right)$. Property 3 follows directly from variation boundedness in the same manner as before since

$$
\begin{aligned}
& \left\|v_{\delta}(x, t+\tau)-v_{\delta}(x, t)\right\|_{L^{1}} \\
& \quad \leqslant \sum_{i=N}^{M-1}\left\|v_{\delta}\left(x, t^{i+1}\right)-v_{\delta}\left(x, t^{i}\right)\right\|_{L^{1}}+\left\|v_{\delta}\left(x, t^{M}\right)-v_{\delta}(x, t+\tau)\right\|_{L^{1}} \\
& \quad \leqslant C(|\tau|+\delta) .
\end{aligned}
$$

So we see there are no difficulties extending the results of Section 4.1 to the fully implicit difference scheme.

For the explicit finite difference scheme we restrict ourselves to one space dimension to obtain the estimates of Proposition 3.1. The extension to higher dimensions is similar to the proofs in Section 4.1. The proofs of the maximum principle, continuity in time and weak limit solution are routine and will be omitted. $L^{1}$ boundedness and entropy satisfying limit solutions follow directly from an inequality of Crandall and Majda [1].

Define

$$
u \vee c=\max (u, c), \quad u \wedge c=\min (u, c) .
$$

Crandall and Majda have shown for any real number $c$

$$
\Delta_{t}^{+}\left|u_{j}^{n}-c\right| \Delta x_{j}+\Delta_{x}^{+} \tilde{F}_{c}(\cdot, \cdot) \Delta t^{n} \leqslant 0
$$

where

$$
\tilde{F}_{c}(\cdot, \cdot)=F\left(u_{j}^{n} \vee c, u_{j-1}^{n} \vee c\right)-F\left(u_{j}^{n} \wedge c, u_{j-1}^{n} \wedge c\right)
$$


To see $\left\{v_{\delta}\right\}$ is bounded in $L^{1}$ sum (4.6) on $j$ and set $c=0$. To see the limit solution in entropy satisfying observe that

$$
\tilde{F}_{c}(u, u)=\operatorname{sgn}(u-c)(f(u)-f(c))
$$

and continue in the manner of the proof of the Lax-Wendroff theorem.

We now need only show the variation remains bounded. Using (2.5) we have

$$
\Delta_{x}^{+} u_{j}^{n+1}=\Delta_{x}^{+}\left(u_{j}^{n}-\lambda_{j} \Delta_{x}^{+} F(\cdot, \cdot)\right) \text {, }
$$

where $\lambda_{j}=\Delta t^{n} / \Delta x_{j}$. Multiply (4.7) by $\psi_{j}^{n+1}$ where $\psi_{j}^{n+1}=\operatorname{sgn}\left(u_{j+1}^{n+1}-u_{j}^{n+1}\right)$ to obtain

$$
\begin{aligned}
\left|\Delta_{x}^{+} u_{j}^{n+1}\right|=\left|\Delta_{x}^{+} u_{j}^{n}\right|+\left(\gamma_{j}-\lambda_{j+1} A_{j+1}+\lambda_{j} B_{j}\right)\left(\psi_{j}^{n+1}-\psi_{j}^{n}\right) \\
-\left(\lambda_{j+1} A_{j+1}+\lambda_{j} B_{j}\right)\left(\psi_{j}^{n+1}-\psi_{j}^{n}\right)-\left(\Delta_{x}^{+} \lambda_{j}\left(A_{j}+B_{j}\right)\right) \psi_{j}^{n},
\end{aligned}
$$

where

$$
\begin{gathered}
A_{j}=F\left(u_{j+1}^{n}, u_{j}^{n}\right)-F\left(u_{j}^{n}, u_{j}^{n}\right), \quad B_{j}=F\left(u_{j}^{n}, u_{j}^{n}\right)-F\left(u_{j}^{n}, u_{j-1}^{n}\right), \\
\gamma_{j}=\left(u_{j+1}^{n}-u_{j}^{n}\right)+\lambda_{j} A_{j}-\lambda_{j+1} B_{j+1} .
\end{gathered}
$$

Summing on $j,(4.8)$ becomes

$$
\begin{aligned}
\sum_{j}\left|\Delta_{x}^{+} u_{j}^{n+1}\right|= & \sum\left|\Delta_{x}^{+} u_{j}^{n}\right|+\sum_{j} \gamma_{j}\left(\psi_{j}^{n+1}-\psi_{j}^{n}\right)+\sum_{j} \lambda_{j} A_{j}\left(\psi_{j}^{n}-\psi_{j-1}^{n+1}\right) \\
& +\sum_{j} \lambda_{j} B_{j}\left(\psi_{j}^{n+1}-\psi_{j-1}^{n}\right) .
\end{aligned}
$$

The last two terms on the right-hand side of (4.9) are nonpositive by 1 and 2 of (2.2). Also

$$
\begin{aligned}
\gamma_{j} \psi_{j}^{n} & =\left|u_{j+1}^{n}-u_{j}^{n}\right|-\lambda_{j}\left|A_{j}\right|-\lambda_{j+1}\left|B_{j+1}\right| \\
& \geqslant\left|u_{j+1}^{n}-u_{j}^{n}\right|-\Lambda H^{u_{j+1}^{n}, u_{j}^{n}}\left(u_{j+1}^{n}, u_{j}^{n}\right) \geqslant 0,
\end{aligned}
$$

so we have $\gamma_{j}\left(\psi_{j}^{n+1}-\psi_{j}^{n}\right) \leqslant 0$. We conclude that

$$
\sum_{j}\left|\Delta_{x}^{+} u_{j}^{n+1}\right| \leqslant \sum_{j}\left|\Delta_{x}^{+} u_{j}^{n}\right|
$$

This estimate together with Lemmas 3.3 and 3.4 completes the proof.

4.3 Proof of Theorem IV. In this section we state and offer an alternate proof of a little known theorem of Kuznetsov [6]. We then apply this result to the difference scheme of Theorem III. The application of Kuznetsov's result to the schemes of Theorems I and II follows in a similar manner.

Lemma 4.1. Let $\omega \in H_{1}^{1}\left(\mathbf{R}^{d} \times \mathbf{R}\right)$. Suppose the support of $\omega$ is contained in $\{(x, t)$ : $|x|<1,|t|<1\}$. Define

$$
\omega_{R, T}=\frac{1}{T R^{d}} \omega\left(\frac{x}{R}, \frac{t}{T}\right)
$$

Then

$$
\left\|\omega_{R, T}\right\|_{L_{1}^{1}\left(\mathbf{R}^{d} \times \mathbf{R}\right)} \leqslant\left(\frac{1}{T}+\frac{1}{R}\right)\|\omega\|_{L_{1}^{1}\left(\mathbf{R}^{d} \times \mathbf{R}\right)} .
$$


Proof. Using the definition of $\omega_{R, T}$ and the change of variables $t / T \rightarrow t^{\prime}$ and $x / R \rightarrow x^{\prime}$ we have

$$
\left\|\omega_{R, T}\right\|_{L_{1}^{1}}=\int_{\mathbf{R}^{d} \times \mathbf{R}}\left(\frac{1}{T}\left|\frac{\partial \omega}{\partial t^{\prime}}\right|+\frac{1}{R}\left|\frac{\partial \omega}{\partial x_{i}^{\prime}}\right|\right) d x^{\prime} d t^{\prime} \leqslant \max \left(\frac{1}{T}, \frac{1}{R}\right)\|\omega\|_{L_{1}^{1}},
$$

from which the desired result is obvious.

Proposition 4.1 (KuZnetsov). Let $v_{\delta}(x, t)$ be an approximate solution of (1.1) satisfying the stability conditions of Proposition 3.1, (conditions 1 through 4). If for all nonnegative and symmetric $\omega\left(x-x^{\prime}, t-t^{\prime}\right) \in C_{0}^{\infty}\left(\mathbf{R}^{d} \times \mathbf{R}\right)$ and all bounded $g\left(x^{\prime}, t^{\prime}\right)$ $\in L^{\infty}\left(L^{1}\left(\mathbf{R}^{d}\right) ;[0, T]\right)$ we have

$$
\begin{aligned}
& -\int_{s}\left|v_{\delta}-g\right| \omega_{t}+\operatorname{sgn}\left(v_{\delta}-g\right)\left(f_{i}\left(v_{\delta}\right)-f_{i}(g)\right) \omega_{x_{i}} d s \\
& \quad \leqslant c\left|t_{1}-t_{0}\right| \delta\|\omega\|_{L_{1}^{1}\left(\mathbf{R}^{d} \times \mathbf{R}\right)}, \quad s=\left(\mathbf{R}^{d} \times\left[t_{0}, t_{1}\right]\right)^{2}, d s=d x d t d x^{\prime} d t^{\prime},
\end{aligned}
$$

then for $T \geqslant t_{1} \geqslant t_{0} \geqslant 0$,

$$
\left\|u\left(x, t_{1}\right)-v_{\delta}\left(x, t_{1}\right)\right\|_{L^{\prime}\left(\mathbf{R}^{d}\right)} \leqslant\left\|u\left(x, t_{0}\right)-v_{\delta}\left(x, t_{0}\right)\right\|_{L^{1}\left(\mathbf{R}^{d}\right)}+K \sqrt{\delta},
$$

where $u(x, t)$ is the unique entropy satisfying solution of (1.1). $K$ depends linearly on the variation of the initial data and $\left|t_{1}-t_{0}\right|$.

Proof. Let $u\left(x^{\prime}, t^{\prime}\right)$ be the unique entropy satisfying solution of $(1.1)$. Let $v_{\delta}(x, t)$ be an approximate solution satisfying conditions 1 through 4 of Proposition 3.1 as well as satisfying (4.10). Consider

$$
I=-\int_{s}\left[\left|u-v_{\delta}\right|\left(\varphi_{t}+\varphi_{t^{\prime}}\right)+F_{i u}\left(v_{\delta}\right)\left(\varphi_{x_{i}}+\varphi_{x_{i}^{\prime}}\right)\right] d s,
$$

where

$$
\begin{gathered}
F_{i u}\left(v_{\delta}\right)=\operatorname{sgn}\left(v_{\delta}-u\right)\left(f_{i}\left(v_{\delta}\right)-f_{i}(u)\right), \\
s=\left(\mathbf{R}^{d} \times \mathbf{R}^{+}\right)^{2}, d s=d x d t d x^{\prime} d t^{\prime} .
\end{gathered}
$$

If $\varphi$ is defined by $\beta\left(t, t^{\prime}\right) \omega\left(x-x^{\prime}, t-t^{\prime}\right)$, where $\beta$ and $\omega$ are smooth, nonnegative functions, $I$ becomes

$$
\begin{aligned}
& -\int_{s}\left[\left|u-v_{\delta}\right| \varphi_{t^{\prime}}+F_{i v_{\delta}}(u) \varphi_{x_{i}^{\prime}}\right] d s, \\
& -\int_{s} \beta\left[\left|u-v_{\delta}\right| \omega_{t}+F_{i u}\left(v_{\delta}\right) \omega_{x_{i}}\right] d s, \\
& -\int_{s}\left|u-v_{\delta}\right| \beta_{t} \omega d s .
\end{aligned}
$$

Since $u\left(x^{\prime}, t^{\prime}\right)$ is an entropy satisfying solution, (1.3) shows the first term above is nonpositive. Therefore

$$
I \leqslant-\int_{s} \beta\left[\left|u-v_{\delta}\right| \omega_{t}+F_{i u}\left(v_{\delta}\right) \omega_{x_{i}}\right] d s-\int_{s}\left|u-v_{\delta}\right| \beta_{t} \omega d s
$$

Also, for this choice of $\varphi$,

$$
\varphi_{x_{i}}+\varphi_{x_{i}^{\prime}}=0, \quad \omega_{t}+\omega_{t^{\prime}}=0
$$


which shows

$$
I=-\int_{s}\left|u-v_{\delta}\right|\left(\beta_{t}+\beta_{t^{\prime}}\right) \omega d s .
$$

Combining (4.12) and (4.13) yields

$$
-\int_{s}\left|u-v_{\delta}\right| \beta_{t^{\prime}} \omega d s \leqslant-\int_{s} \beta\left[\left|u-v_{\delta}\right| \omega_{t}+F_{i u}\left(v_{\delta}\right) \omega_{x_{i}}\right] d s
$$

Now let $\theta$ be a nonnegative, symmetric, $C_{0}^{\infty}\left(\mathbf{R}^{+}\right)$function having unit mass. Define

$$
\theta_{\varepsilon}(t)=\frac{1}{\varepsilon} \theta\left(\frac{t}{\varepsilon}\right)
$$

and

$$
\chi_{\left[t_{0}, t_{1}\right]}(t)= \begin{cases}1, & t \in\left[t_{0}, t_{1}\right] \\ 0, & \text { otherwise }\end{cases}
$$

Let $\chi_{\varepsilon}(t)$ be defined by the convolution

$$
\chi_{\varepsilon}(t)=\left(\chi_{\left[t_{0}, t_{1}\right]} * \theta_{\varepsilon}\right)(t)
$$

It is easily shown that $\chi_{\varepsilon}(t) \geqslant 0, \chi_{\varepsilon}(t) \in C_{0}^{\infty}\left(\mathbf{R}^{+}\right)$and

$$
\frac{d}{d t} \chi_{\varepsilon}(t)=\theta_{\varepsilon}\left(t-t_{0}\right)-\theta_{\varepsilon}\left(t-t_{1}\right) .
$$

In equality (4.14) set

$$
\beta\left(t, t^{\prime}\right)=\chi_{\varepsilon}(t) \chi_{\varepsilon}\left(t^{\prime}\right)
$$

Sending $\varepsilon$ to $0,(4.14)$ becomes

$$
\begin{aligned}
\int_{s_{2}}\left|u\left(x^{\prime}, t_{1}\right)-v_{\delta}(x, t)\right| \omega\left(x-x^{\prime}, t-t_{1}\right) d s_{2} \\
-\int_{s_{2}}\left|u\left(x^{\prime}, t_{0}\right)-v_{\delta}(x, t)\right| \omega\left(x-x^{\prime}, t-t_{0}\right) d s_{2} \leqslant E
\end{aligned}
$$

where

$$
s_{2}=\mathbf{R}^{d} \times\left(\mathbf{R}^{d} \times\left[t_{0}, t_{1}\right]\right), \quad d s_{2}=d x d x^{\prime} d t
$$

and

$$
E=\int_{t_{0}}^{t_{1}} \int_{t_{0}}^{t_{1}} \int_{\mathbf{R}^{d} \times \mathbf{R}^{d}}\left[\left|u-v_{\delta}\right| \omega_{t}+F_{i u}\left(v_{\delta}\right) \omega_{x_{i}}\right] d s
$$

Now let $\omega$ be a nonnegative, symmetric, $C_{0}^{\infty}\left(\mathbf{R}^{d} \times \mathbf{R}\right)$ function having unit mass. Further suppose that

$$
\operatorname{support}(\omega) \subseteq(-R, R) \times(-T, T),
$$


where $T \leqslant\left(t_{1}-t_{0}\right)$. It is not hard to show that

$$
\begin{aligned}
& \left|\int_{s_{2}}\right| u\left(x^{\prime}, t_{0,1}\right)-v_{\delta}(x, t) \mid \omega\left(x-x^{\prime}, t-t_{0,1}\right) d s_{2} \\
& -\frac{1}{2} \int_{\mathbf{R}^{d}}\left|u\left(x, t_{0,1}\right)-v_{\delta}\left(x, t_{0,1}\right)\right| d x \mid \\
& \leqslant \sup _{t} \sup _{|h|<R} \int_{\mathbf{R}^{d}}|u(x+h, t)-u(x, t)| d x \\
& +\sup \sup _{t} \int_{0<\tau<T}\left|v_{\mathbf{R}^{d}}(x, t+\tau)-v_{\delta}(x, t)\right| d x \\
& \equiv \lambda_{x}(u, R)+\lambda_{t}\left(v_{\delta}, T\right) \text {. }
\end{aligned}
$$

This inequality along with inequality (4.15) gives

$$
\begin{aligned}
\left\|u\left(x, t_{1}\right)-v_{\delta}\left(x, t_{1}\right)\right\|_{L^{1}\left(\mathbf{R}^{d}\right)}-\left\|u\left(x, t_{0}\right)-v_{\delta}\left(x, t_{0}\right)\right\|_{L^{1}\left(\mathbf{R}^{d}\right)} \\
\\
\quad \leqslant \lambda_{x}(u, R)+\lambda_{t}\left(v_{\delta}, T\right)+E / 2 .
\end{aligned}
$$

Conditions 2 and 3 of Proposition 3.1 show that

$$
\lambda_{x}(u, R) \leqslant K_{2} R, \quad \lambda_{t}\left(v_{\delta}, T\right) \leqslant K_{3}(T+\delta)
$$

and (4.10) required that

$$
E \leqslant c\left|t_{1}-t_{0}\right| \delta\|\omega\|_{L_{1}^{1}\left(\mathbf{R}^{d} \times \mathbf{R}\right)} .
$$

Therefore, with the aid of Lemma 4.1 we see that the right-hand side of (4.16) is bounded by

$$
K[R+\delta / R+T+\delta / T]
$$

Minimizing this quantity with respect to $R$ and $T$ gives the required result.

To obtain (4.10) of Proposition 4.1 the approximate solution must satisfy an approximate entropy condition. This will become evident in the proof of Theorem IV.

We need one further simple lemma.

LEMMA 4.2. Let $\omega \in H_{1}^{1}\left(\mathbf{R}^{d} \times \mathbf{R}\right)$. Then

$$
\|\omega(x+h, t+\tau)-\omega(x, t)\|_{L^{1}\left(\mathbf{R}^{d} \times \mathbf{R}\right)} \leqslant(d|h|+|\tau|)\|\omega\|_{L_{1}^{1}\left(\mathbf{R}^{d} \times \mathbf{R}\right)} .
$$

Proof. By the completeness of $H_{1}^{1}$ we may assume $\omega \in C_{0}^{\infty}\left(\mathbf{R}^{d} \times \mathbf{R}\right)$, in which case

$$
\omega(x, t+\tau)-\omega(x, t)=\int_{0}^{\tau} \frac{\partial \omega}{\partial t^{\prime}}\left(x, t+t^{\prime}\right) d t^{\prime}
$$

and

$$
\omega\left(x+h_{i}, t\right)-\omega(x, t)=\int_{0}^{h_{i}} \frac{\partial \omega}{\partial x_{i}^{\prime}}\left(x+x^{\prime}, t\right) d x^{\prime} .
$$

This identity, the triangle inequality and exchanging the order of integration shows that

$$
\|\omega(x+h, t+\tau)-\omega(x, t)\|_{L^{1}} \leqslant \int_{0}^{|\tau|}\left\|\frac{\partial \omega}{\partial t}\right\|_{L^{1}} d t^{\prime}+\int_{0}^{\mid h_{i}}\left\|\frac{\partial \omega}{\partial x_{i}}\right\|_{L^{1}} d x_{i}^{\prime} .
$$

The final estimate is now easily seen. 
Proof of Theorem IV. Let $v_{\delta}(x, t)$ be obtained from an explicit finite difference scheme of the form (2.5) and suppose $v_{\delta}$ satisfies the approximate entropy condition (4.6). Then $v_{\delta}$ satisfies condition (4.10) of Proposition 4.1.

We again lose no generality assuming $d=1$. Let $v_{\delta}$ be a function of $x, t$ and let $g$ be a function of $x^{\prime}, t^{\prime}$. Define

$$
F_{g}\left(v_{\delta}\right)=\operatorname{sgn}\left(v_{\delta}-g\right)\left(f\left(v_{\delta}\right)-f(g)\right)
$$

Since $v_{\delta}(x, t)$ is piecewise constant

$$
\begin{aligned}
-\int_{s \times s}\left[\left|v_{\delta}-g\right| \omega_{t}\right. & \left.+F_{g}\left(v_{\delta}\right) \omega_{x}\right] d s \\
& =-\int_{s}\left[\sum_{j, n} \int_{\Omega_{j}^{n}}\left[\left|u_{j}^{n}-g\right| \omega_{t}+F_{g}\left(u_{j}^{n}\right) \omega_{x}\right] d x d t\right] d x^{\prime} d t^{\prime},
\end{aligned}
$$

where $s=\mathbf{R} \times\left[t_{0}, t_{1}\right]$ and $d s=d x d t d x^{\prime} d t^{\prime}$. Integrating and applying the integral version of the mean value theorem along with summing by parts shows the right-hand side of (4.17) is equal to

$$
\begin{aligned}
& \int_{s\left[\sum_{j, n} \Delta_{t}^{+}\left|u_{j}^{n}-g\right| \omega\left(\xi_{j}-x^{\prime}, t^{n+1}-t^{\prime}\right) \Delta x_{j}\right.} \\
& \left.\quad+\Delta_{x}^{+} F_{g}\left(u_{j}^{n}\right) \omega\left(x_{j+1}-x^{\prime}, \tau^{n}-t^{\prime}\right) \Delta t^{n}\right] d x^{\prime} d t^{\prime},
\end{aligned}
$$

where $\xi_{j} \in\left[x_{j}, x_{j+1}\right]$ and $\tau^{n} \in\left[t^{n}, t^{n+1}\right]$. Adding and subtracting

$$
\omega\left(x_{j+1}-x^{\prime}, \tau^{n}-t^{\prime}\right) \text {, }
$$

this becomes

$$
\begin{gathered}
\int_{s, n} \sum_{j, n} \Delta x_{j}\left[\Delta _ { t } ^ { + } | u _ { j } ^ { n } - g | \left(\omega\left(\xi_{j}-x^{\prime}, t^{n+1}-t^{\prime}\right)\right.\right. \\
\left.\left.-\omega\left(x_{j+1}-x^{\prime}, \tau^{n}-t^{\prime}\right)\right)\right] d x^{\prime} d t^{\prime} \\
+\int_{s} \sum_{j, n}\left[\Delta_{i}^{+}\left|u_{j}^{n}-g\right| \Delta x_{j}+\Delta_{x}^{+} F_{j}\left(u_{j}^{n}\right) \Delta t^{n}\right] \omega\left(x_{j+1}-x^{\prime}, \tau^{n}-t^{\prime}\right) d x^{\prime} d t^{\prime}
\end{gathered}
$$

The triangle inequality shows that the first term of (4.18) is bounded by

$$
\sum_{j, n} \Delta x_{j}\left|\Delta_{t}^{+} u_{j}^{n}\right| \int_{s}\left|\omega\left(x^{\prime}-\xi_{j}, t^{\prime}-t^{n+1}\right)-\omega\left(x^{\prime}-x_{j+1}, t^{\prime}-\tau^{n}\right)\right| d x^{\prime} d t^{\prime} .
$$

Lemma 4.2 along with the continuity in time estimate, (condition 3 of Proposition 3.1), shows this quantity is bounded by

$$
K_{3}\left(\left|t_{1}-t_{0}\right|+\delta\right) \cdot 2 \delta\|\omega\|_{L_{1}^{1}(\mathbf{R} \times \mathbf{R})} .
$$

To estimate the second term of (4.18) recall the definition of $\tilde{F}_{c}(\cdot, \cdot)$; see Eq. (4.6). Adding and subtracting this, the second term of (4.18) becomes

$$
\begin{aligned}
& \int_{s_{j, n}} \sum_{t}\left[\Delta_{t}^{+}\left|u_{j}^{n}-g\right| \Delta x_{j}+\Delta_{x}^{+} \tilde{F}_{g}\left(u_{j}^{n}, u_{j-1}^{n}\right) \Delta t^{n}\right] \cdot \omega\left(x^{\prime}-x_{j+1}, t^{\prime}-\tau^{n}\right) d x d t \\
& \quad+\int_{s} \sum_{j, n} \Delta t^{n} \Delta_{x}^{+}\left(\tilde{F}_{g}\left(u_{j}^{n}, u_{j}^{n}\right)-\tilde{F}_{g}\left(u_{j}^{n}, u_{j-1}^{n}\right)\right) \cdot \omega\left(x^{\prime}-x_{j+1}, t^{\prime}-\tau^{n}\right) d x d t .
\end{aligned}
$$


Inequality (4.6) shows the first term above is nonpositive. Using the fact that the variation of $v_{\delta}$ is bounded, we can bound the second term of (4.20) by

$$
\begin{aligned}
\int_{s, n} \Delta t^{n}\left|\tilde{F}_{g}\left(u_{j}^{n}, u_{j}^{n}\right)-\tilde{F}_{g}\left(u_{j}^{n}, u_{j-1}^{n}\right)\right| \\
\cdot\left|\omega\left(x^{\prime}-x_{j}, t^{\prime}-\tau^{n}\right)-\omega\left(x^{\prime}-x_{j+1}, t^{\prime}-\tau^{n}\right)\right| d x d t \\
\leqslant 2 K \cdot K_{2}\left|t_{1}-t_{0}\right| \cdot \delta\|\omega\|_{L_{1}^{1}(\mathbf{R} \times \mathbf{R})} .
\end{aligned}
$$

Combining (4.19) and (4.21) completes the proof.

We see the crucial part of the proof of Theorem IV was the utilization of the approximate entropy condition in Eq. (4.20). The other schemes discussed also have approximate entropy conditions; see Eq. (4.5). Therefore this result extends to the method of lines and the implicit finite difference scheme in a similar manner.

Appendix I. Definitions of Spaces and Norms. 1. The space $C_{0}^{P}\left(\mathbf{R}^{d}\right)$ is defined to be the space of all compactly supported functions that have $P$ continuous derivatives.

2. The space $L^{1}\left(\mathbf{R}^{d}\right)$ is the completion of $C_{0}^{\infty}\left(\mathbf{R}^{d}\right)$ under the $L^{1}$ norm.

3. The $L_{1}^{1}\left(\mathbf{R}^{d} \times \mathbf{R}\right)$ seminorm is defined as

$$
\|f\|_{L_{1}^{1}\left(\mathbf{R}^{d} \times \mathbf{R}\right)}=\left\|\frac{\partial f}{\partial t}\right\|_{L^{1}\left(\mathbf{R}^{d} \times \mathbf{R}\right)}+\left\|\frac{\partial f}{\partial x_{i}}\right\|_{L^{\prime}\left(\mathbf{R}^{d} \times \mathbf{R}\right)} .
$$

4. The space $H_{1}^{1}\left(\mathbf{R}^{d} \times \mathbf{R}\right)$ is the completion of $C_{0}^{\infty}\left(\mathbf{R}^{d} \times \mathbf{R}\right)$ under the norm

$$
\|f\|_{H_{1}^{1}\left(\mathbf{R}^{d} \times \mathbf{R}\right)}=\|f\|_{L_{1}^{1}\left(\mathbf{R}^{d} \times \mathbf{R}\right)}+\|f\|_{L^{1}\left(\mathbf{R}^{d} \times \mathbf{R}\right)} \text {. }
$$

5. The variation of a real-valued function defined on $\mathbf{R}^{2}$ is defined by

$$
\operatorname{Var}(f(x, y))=\int_{\mathbf{R}} \operatorname{Var}_{x}(f(x, y)) d y+\int_{\mathbf{R}} \operatorname{Var}_{y}(f(x, y)) d x
$$

where $\operatorname{Var}_{x} f(x, y)$ is the usual variation of $f(\cdot, y)$. The extension of this definition to higher dimensions is obvious.

\section{Appendix II.}

Proof of Lemma 3.1. Let $\left\{s_{k}\right\}$ be a countable dense subset of $[0, T]$. Inequalities 1 and 2 show there exists a subsequence of $\left\{v_{\delta}\right\}$, say $\left\{v_{\delta_{1}}\right\}$, such that $v_{\delta_{1}}\left(x, s_{1}\right) \rightarrow$ $v\left(x, s_{1}\right)$ in $L_{\text {loc }}^{1}\left(\mathbf{R}^{d}\right)$; see [2]. Further, a Cantor diagonalization argument gives us a subsequence that converges at every $s_{k}$. For convenience, call this subsequence $\left\{v_{\delta}\right\}$. This is the required sequence.

Partition $[0, T]$ into $N$ intervals $\left(t_{i}, t_{i+1}\right)$, where the $t_{i}$ 's are selected from $\left\{s_{k}\right\}$ and each interval has length less than $\varepsilon$. From above we see that $\left\{v_{\delta}\right\}$ is Cauchy at each $t_{i}$, so

$$
\left\|v_{\delta_{1}}\left(x, t_{i}\right)-v_{\delta_{2}}\left(x, t_{i}\right)\right\|<\varepsilon \quad \forall \delta_{1}, \delta_{2}<\Delta\left(t_{i}\right)
$$

Let $\Delta=\min \left(\Delta\left(t_{1}\right), \ldots, \Delta\left(t_{N}\right)\right)$. Using 3 of Proposition 3.1 we have for any $t$ in $[0, T]$

$$
\begin{aligned}
\| v_{\delta_{1}}(x, t)- & v_{\delta_{2}}(x, t) \|_{L^{1}} \\
\leqslant & \left\|v_{\delta_{1}}(x, t)-v_{\delta_{1}}\left(x, t_{i}\right)\right\|_{L^{1}}+\left\|v_{\delta_{1}}\left(x, t_{i}\right)-v_{\delta_{2}}\left(x, t_{i}\right)\right\|_{L^{1}} \\
& \quad+\left\|v_{\delta_{2}}(x, t)-v_{\delta_{2}}\left(x, t_{i}\right)\right\|_{L^{1}} \\
\leqslant & K_{3}\left[2\left|t-t_{i}\right|+\delta_{1}+\delta_{2}\right]+\varepsilon .
\end{aligned}
$$

Choosing $\delta_{1}, \delta_{2}<\min (\Delta, \varepsilon)$ and $\left|t_{i}-t\right|<\varepsilon$ completes the proof. 
Proof of Lemma 3.2. Since $v_{\delta_{k}}$ converges boundedly in $L_{\text {loc }}^{1}$, uniformly in the strip $[0, T]$, there exists a bounded subsequence that converges pointwise a.e. to $v . \varphi$ is compactly supported, therefore the bounded convergence theorem along with 5 of Proposition 3.1 shows $v$ is a weak solution. We also have, using 1 , that

$$
\begin{aligned}
& \left\|v(x, t)-u_{0}(x)\right\|_{L^{1}} \\
& \quad \leqslant\left\|v(x, t)-v_{\delta}(x, t)\right\|_{L^{1}}+\left\|v_{\delta}(x, t)-v_{\delta}(x, 0)\right\|_{L^{1}}+\left\|v_{\delta}(x, 0)-u_{0}(x)\right\|_{L^{1}} \\
& \quad \leqslant \varepsilon+K_{3}(|t|+\delta)+\varepsilon,
\end{aligned}
$$

which shows

$$
\lim _{t \downarrow 0}\left\|v(x, t)-u_{0}(x)\right\|_{L^{1}}=0
$$

Department of Mathematics

University of Southern California

Los Angeles, California 90007

1. M. Crandall \& A. Majda, "Monotone difference approximations for scalar conservation laws," Math. Comp., v. 34, 1980, pp. 1-22.

2. N. DUNford \& J. SCHWARTz, Linear Operators, Part 1: General Theory, Pure and Appl. Math., vol. VII, Interscience, New York, 1958.

3. B. ENGQUist \& S. Osher, "Stable and entropy satisfying approximations for transonic flow calculations," Math. Comp., v. 34, 1980, pp. 45-75.

4. A. Harten, J. M. Hyman \& P. D. Lax, "On finite difference approximations and entropy conditions for shocks," Comm. Pure Appl. Math., v. 29, 1976, pp. 297-322.

5. S. N. KRUŽxov, "First order quasi-linear equations with several space variables," Math. USSR Sb., v. 10, 1970, pp. $217-243$.

6. N. N. KuZNETSOv, "On stable methods for solving non-linear first order partial differential equations in the class of discontinuous functions," Topics in Numerical Analysis, III (Proc. Roy. Irish Acad. Conf., Trinity Coll., Dublin, 1976), pp. 183-197.

7. P. D. LAX, "Hyperbolic systems of conservation laws and the mathematical theory of shock waves," SIAM Reg. Conf. Series in Appl. Math., v. 11, 1972.

8. P. D. LaX \& B. Wendroff, "Systems of conservation laws," Comm. Pure Appl. Math., v. 13, 1960, pp. 217-237. 